\title{
„ER SOLL AN DER ORGANISATION \\ FESTHALTEN": ZUM WORTLAUT VON LASSALLES TESTAMENT
}

Lassalles Testament ist das entscheidende Zeugnis dafür, wie ganz persönlich Lassalle den ADAV als seine eigenste Schöpfung auffaßte, wie sehr er die Organisation mit seiner Person identifizierte. Obwohl der Lassalle unwandelbar wohlgesinnte Franz Mehring die von Lassalle ausgesetzten Legate und Renten als dem Parteiinteresse dienend interpretierte, konnte er doch nicht umhin, das Testament als verhängnisvoll für die Weiterentwicklung des ADAV zu bezeichnen. Im zweiten Kapitel des vierten Buches seiner Geschichte der deutschen Sozialdemokratie findet sich dazu die folgende Passage: „Verhängnisvoller erwiesen sich die Bestimmungen des Testaments, die sich unmittelbar auf den Allgemeinen Deutschen Arbeiterverein bezogen. Lassalle empfahl dem Vereine, den Frankfurter Bevollmächtigten Bernhard Becker zu seinem Nachfolger zu wählen, und diesem band er auf die Seele, an der Organisation festzuhalten, die den Verein zum Siege führen werde, Verfügungen, die sich als Reflexe der Vahlteichschen Opposition erklären, aber, statt alte Verwirrung zu schlichten, nur neue Verwirrung angerichtet haben."1

Die Lassalleaner waren als Organisationsfanatiker bekannt und gefürchtet Lassalles Vermächtnis ,an der Organisation festzuhalten”, weil sie allein den Sieg garantiere, wurde zum Glaubensartikel, zugleich aber auch, wie Mehring richtig feststellte, eine Quelle anhaltender Verwirrung. Die Fraktionskämpfe im ADAV und anschließend daran die Parteikämpfe in der Arbeiterbewegung lassen sich anhand von Mehrings Darstellung genau verfolgen, ${ }^{2}$ und wenn diese auch keineswegs in Lassalles Testament allein ihre Ursache haben und eine soziale wie historische Erklärung verlangen, so kommt doch Lassalles „Ruf aus dem Grabe” eine bestimmte und bestimmende Bedeutung zu.

An der Authentizität jenes Rufes ist nie gezweifelt worden, findet

1 Siehe Franz Mehring, Gesammelte Schriften, Band 2, Berlin 1960, S. 171.

2 Ebd., S. 181ff. 
sich doch der volle Wortlaut des Testaments in den verschiedenen Gesamtausgaben von Lassalles Werken, wie letztlich in der Bernsteinschen Ausgabe von 1919:

„Dem Allgemeinen Deutschen Arbeiterverein empfehle ich, zu meinem Nachfolger den Frankfurter Bevollmächtigten Bernhard Becker zu wählen. Er soll an der Organisation festhalten! Sie wird den Arbeiterstand zum Sieg führen."1

Und doch ist dieser Text keineswegs gesichert.

In Otto Dammers Rundschreiben an alle „Bevollmächtigten” des ADAV, das er in seiner Eigenschaft als amtierender Präsident am 15. Oktober 1864 erließ (,,Verfügung an sämmtliche Bevollmächtigte des Allgemeinen Deutschen Arbeiter-Vereins"), ${ }^{2}$ heißt es an der einschlägigen Stelle:

„Ich habe nur deshalb bis heute [mit der Neuwahl eines Präsidenten] gezögert, weil ich den Gemeinden zuverlässige Nachricht geben wollte von dem letzten Willen unsers großen Todten, welchen er in Bezug auf seinen Nachfolger ausgesprochen hat. Lassalle sagt in seinem Testament nach Mittheilung der Testamentsexecutoren Rechtsanwalt Holthoff und Lothar Bucher in Berlin: ,Dem Allgemeinen Deutschen Arbeiterverein empfehle ich, zu meinem Nachfolger den Frankfurter Bevollmächtigten Bernhard Becker zu wählen. Er soll die Organisation festhalten; sie wird den Arbeiterstand zum Siege führen."'

Es sei sogleich festgestellt, daß der Unterschied zwischen der von Bernstein adoptierten Fassung und der von Dammer nach dem Zitat der Holthoff-Bucherschen Nachricht in dem einzigen Wort „an” liegt, strittig also nur ist, ob die Aufforderung an Becker lautet, "die Organisation" festzuhalten, oder ,an der Organisation”.

Vor der Analyse dieses Unterschiedes ist noch auf einen Brief der Gräfin Sophie v. Hatzfeldt an Otto Dammer, den amtierenden Präsidenten des ADAV, vom 13. Oktober 1864 zu verweisen, den Hermann Oncken zusammen mit einem anderen vom 6. Oktober von Dammer erhalten hatte und im siebenten Band von Grünbergs Archiv veröffentlichte. ${ }^{3}$ Die Briefe behandeln die Krisenzeit nach Lassalles Tod und die Anwartschaft Beckers auf den Posten des Präsidenten. Die

1 Ferdinand Lassalle, Gesammelte Reden und Schriften, hrsg. von Eduard Bernstein, IV, Berlin 1919, S. 338.

2 Nach der Kopie im Herwegh-Archiv, Liestal, Nr 1492.

${ }^{3}$ „Aus dem Streit um Lassalles Erbe. Zwei Briefe der Gräfin Hatzfeldt aus dem Oktober 1864", mitgeteilt von Hermann Oncken, in: Grünbergs Archiv, VII (1916), S. 95ff. Merkwürdigerweise nennt Oncken den Adressaten Udo Dammer. 
Gräfin beruft sich auf die entsprechende Stelle aus Lassalles Testament und wir hören genau wie in Dammers Erlaß von einem „Festhalten der Organisation” und keineswegs von einem „Festhalten an der Organisation". Dammers Fassung bekommt dadurch erhöhte Authentizität. Die Gräfin schreibt an Dammer am 13. Oktober:

„Soeben trifft das Testament Lassalles hier ein und ich beeile mich, Ihnen die betreffenden Worte über $B$. Becker hier wörtlich mitzuteilen.

,Dem Allg. D. Arbeiter Verein empfehle ich zu meinem Nachfolger den Frankfurter Bevollmächtigten B. Becker zu wählen. Er soll die Organisation festhalten! Sie wird den Arbeiterstand zum Siege führen'."1

Es ist natürlich außerordentlich wichtig, daß die Gräfin die Genauigkeit ihrer Abschrift, mit dem Originaltestament vor Augen, betont; auch ist ihr Wille zur Genauigkeit nicht anzuzweifeln, da es ihr zwar nicht um die Nuance ging, die hier hervorgehoben wird, sondern um den Beweis, Lassalle habe Becker nicht irgendwie „empfohlen”, sondern seine Wahl testamentarisch angeordnet.

Wenn also Dammer in seinem Rundschreiben das „Festhalten der Organisation" im Namen des Verstorbenen verlangt, hat er sich auf zwei von einander unabhängige und durchaus glaubwürdige Informationsquellen berufen; sollte er sich dennoch im Irrtum befunden haben - was natürlich nicht ausgeschlossen ist - , so müßte das sehr verwundern.

$\mathrm{Zu}$ erörtern bleibt die Frage, wie wichtig der Unterschied der beiden Fassungen zu nehmen ist. Es scheint, als gäbe darüber der Brief der Gräfin an Dammer vom 6. Oktober ${ }^{2}$ Aufschluß. Die Gräfin schreibt nach einigen einleitenden Worten:

„Sie fragen B. Becker, woher er weiss, dass Ferdinand Lassalle ihn zu seinem Nachfolger bestimmt habe.

Er weiss es von mir. Lassalle hat mir schon in Frankfurt, wo wir in den ersten Tagen [im] Juli zusammen waren, seinen Entschluss mitgeteilt, falls er beseitigt werden sollte, die Präsidentur $B$. Becker zu übertragen, indem er hinzusetzte, dass die énergie Beckers ihm Bürgschaft gebe, dass er dieselbe organisation und feste discipline, die er eingeführt, unverändert fortsetzen würde, und das halte er für die erste Lebensbedingung des Vereins."

Die beiden französischen Synonyme organisation und discipline sind

1 Ebd., S. 97.

2 Ebd., S. 95. 
wohl der Schlüssel zum Verständnis von Lassalles letzter Willensäußerung: es geht ihm keineswegs um diesen oder jenen Paragraphen des Statuts oder der Geschäftsordnung, wie später bei den Fraktionskämpfen, sondern um das Prinzip der Zentralisation, das der demissionierte erste Vereinssekretär Julius Vahlteich seit dem Februar 1864 zuerst mehr privat und dann immer mehr auch öffentlich angegriffen hatte. Hinsichtlich der Wahrung des reinen Partei- und Agitationscharakters des ADAV war Bernhard Becker, der aggressive Frankfurter Bevollmächtigte, allerdings der geeignete Mann, hatte er doch in verschiedenen schriftlichen Außerungen klar zu erkennen gegeben, wie sehr er jede "Vereinsspielerei” ablehnte, wie er sich ja auch bei der Bekämpfung der Maingauorganisation der Arbeiterbildungsvereine hervorgetan hatte. ${ }^{1}$ Dem entsprach auch seine Vergangenheit, hatte er doch in der Emigration in England an verschiedenen Geheimbünden teilgenommen. Auch war sein $\mathrm{HaB}$ gegenüber dem von Lassalle verabscheuten Nationalverein und der hinter ihm stehenden preußischen Fortschrittspartei zu radikalstem Ausdruck gekommen. ${ }^{2}$ Es war also sinnvoll, Becker an die Spitze des ADAV zu stellen, wenn jeder Rückfall in das verpönte „Vereinsgebaren” vermieden werden sollte und den ehemaligen Anhängern der Bildungsvereine der „FortschrittsTic"3 auszutreiben war. All das ist mit dem Wort vom „Festhalten der Organisation" gut ausgedrückt, hat aber nichts mit Fetischismus der Statuten zu tun, in deren Zeichen man vom „Festhalten an der Organisation" sprach.

Eine offene Frage muß bleiben, wie der Sprachgebrauch vom „Festhalten an der Organisation" aufkam, wenn er nicht im Testament selbst enthalten war. $\mathrm{Zu}$ denken wäre in erster Hinsicht an die Gräfin, die sich bald von Bernhard Becker abwandte und in seiner Zusammenlegung des Sekretariats mit der Präsidentschaft einen Statutenbruch sah, den sie mit der Formel von Lassalles Vermächtnis bekämpfen wollte. Aber auch Willms, Klings, Karl Bruhn und vielleicht auch Becker selbst möchten versucht gewesen sein, ihre Position als allein mit der zum Heiligtum erklärten Verfassung des ADAV in Übereinstimmung befindlich hinzustellen.

Lassalles testamentarische Bestimmungen lassen sich viel rigoroser kritisieren als Mehring es getan hat. Sowohl im An- wie Aberkennen

1 Vgl. Ferdinand Lassalle, Nachgelassene Briefe und Schriften, hrsg. von Gustav Mayer, V, Stuttgart 1925, S. 254ff., 336ff.

2Vgl. B. Becker, Die Geschichte der Arbeiter-Agitation Ferdinand Lassalle's, Braunschweig 1875, S. 11ff., und W. Blos, Denkwürdigkeiten eines Sozialdemokraten, I, München 1914, S. $127 \mathrm{ff}$.

3 Siehe „Briefe Lassalles an Dr. Otto Dammer in Leipzig", in: Grünbergs Archiv, II (1912), S. 415. 
der Verdienste, die er seinem nächsten Bekanntenkreis zuteil werden ließ, beging er unglaubliche Fehler; ${ }^{1}$ was den ADAV betrifft, hat er ihn nicht als einen selbständigen sozialen Organismus behandelt, sondern wie seine Privatdomäne. Von dem größten Fehler ist er aber, wie es scheint, doch freizusprechen: wohl hat er die Zentralisation als Kampfwaffe verteidigt so wie er die Dinge verstand, aber dennoch nicht beabsichtigt, den ADAV für ewig an die Paragraphen eines unheilvollen Statuts zu ketten. So wäre also der zumindest teilweise sicherlich nachzuweisende Sektencharakter des ADAV, wie er sich vor allem im Statutenfetischismus kundtat, ohne direkte Bezugnahme auf Lassalles Testament zu erklären.

1 Vlg. S. Na'aman, Lassalle, Hannover 1970, S. 784ff. 